\title{
Note on the number of semistar operations, XI
}

\author{
Ryûki MATSUDA*
}

\begin{abstract}
We study star operations and semistar operations on an almost pseudovaluation domain.
\end{abstract}

The notion of a star operation is classical, and that of a Kronecker function ring which arises by a star operation is also classical. Let $D$ be an integral domain, let $K$ be its quotient field, and let $\mathrm{F}(D)$ be the set of non-zero fractional ideals of $D$. A mapping $I \longmapsto I^{*}$ from $\mathrm{F}(D)$ to $\mathrm{F}(D)$ is called a star operaion on $D$, if it satisfies the following conditions: (1) $(x)^{*}=(x)$ for each $x \in K-\{0\} ;(2)(x I)^{*}=x I^{*}$ for each $x \in K-\{0\}$ and $I \in \mathrm{F}(D) ;(3) I \subset I^{*}$ for each $I \in \mathrm{F}(D) ;(4)\left(I^{*}\right)^{*}=I^{*}$ for each $I \in \mathrm{F}(D)$; (5) $I \subset J$ implies $I^{*} \subset J^{*}$ for each $I, J \in \mathrm{F}(D)$. The Kronecker function ring of $D$ with respect to a star operation $*$ on $D$ was first defined by Prüfer $([\mathrm{P}])$ and further investigated by Krull $([\mathrm{K}])$. Let $\mathrm{F}^{\prime}(D)$ be the set of non-zero $D$-submodules of $K$. A mapping $I \longmapsto I^{*}$ from $\mathrm{F}^{\prime}(D)$ to $\mathrm{F}^{\prime}(D)$ is called a semistar operation on $D$, if it satisfies the following conditions: (1) $(x I)^{*}=x I^{*}$ for each $x \in K-\{0\}$ and $I \in \mathrm{F}^{\prime}(D)$; (2) $I \subset I^{*}$ for each $I \in \mathrm{F}^{\prime}(D)$; (3) $\left(I^{*}\right)^{*}=I^{*}$ for each $I \in \mathrm{F}^{\prime}(D)$; (4) $I \subset J$ implies $I^{*} \subset J^{*}$ for each $I, J \in \mathrm{F}^{\prime}(D)$. We confer Fontana-Loper $([\mathrm{FL}])$ and Halter-Koch $([\mathrm{HK}])$ for notions of star operations, semistar operations, and their Kronecker function rings.

Let $\Sigma(D)$ (resp. $\Sigma^{\prime}(D)$ ) be the set of star operations (resp. semistar operations) on $D$. In this paper, we are interested in cardinalities $|\Sigma(D)|$ and $\left|\Sigma^{\prime}(D)\right|$.

Let $D$ be an integrally closed domain. Then $D$ has only a finite number of semistar operations if and only if $D$ is a finite dimensional Prüfer domain with only a finite number of maximal ideals ([M4]).

Let $V$ be a valuation domain with dimension $n$, let $v$ be a valuation belonging to $V$, and let $\Gamma$ be its value group. Let $M=P_{n} \supsetneqq P_{n-1} \supsetneqq \cdots \cdots P_{1} \supsetneqq(0)$ be the prime ideals of $V$, and let $\{0\} \varsubsetneqq H_{n-1} \subsetneq \cdots \varsubsetneqq H_{1} \varsubsetneqq \Gamma$ be the convex subgroups of $\Gamma$. Let $m$ be a positive integer with $n+1 \leq m \leq 2 n+1$. Then the following conditions are equivalent: (1) $\left|\Sigma^{\prime}(V)\right|=m$; (2) The maximal ideal of $V_{P_{i}}$ is principal for exactly $2 n+1-m$ of $i$; (3) $\Gamma / H_{i}$ has a minimal positive element for exactly $2 n+1-m$ of $i$ $([\mathrm{M} 1])$.

Received 30 November, 2006; revised 7 March, 2007.

2000 Mathematics Subject Classification. Primary 13A15.

Key Words and Phrases. star opcration, semistar operation, pseudo-valuation domain, almost pseudovaluation domain.

${ }^{*}$ Professor Emeritus, Ibaraki University 
In [M5], we studied star operations and semistar operations on a pseudo-valuation domain (or a PVD) $D$. We gave conditions for $D$ to have only a finite number of semistar operations, and showed that conditions for $\left|\Sigma^{\prime}(D)\right|<\infty$ reduce to conditions for fields.

In this paper, we concern with star operations and semistar operations on almost pseudo-valuation domains. We study almost pseudo-valuation domains with simple associated valuation rings, and will prove the following,

Theorem Let $D$ be an almost pseudo-valuation domain, let $P$ be the maximal ideal of $D$, let $V=(P: P)$, let $M$ be the maximal ideal of $V$, and let $K=V / M$. Assume that the valuation ring $V$ is discrete with rank one and $D / P=K$. Then we have

(1) If $K$ is a finite field, then $|\Sigma(D)|<\infty$.

(2) If $P=M^{2}$, then $|\Sigma(D)|=1$ and $\left|\Sigma^{\prime}(D)\right|=3$, and if $P=M^{3}$, then $|\Sigma(D)|=3$ and $\left|\Sigma^{\prime}(D)\right|=6$.

(3) If $P=M^{4}$ and $K$ is an infinite field, then $|\Sigma(D)|=\infty$ and $\left|\Sigma^{\prime}(D)\right|=\infty$.

(4) If $P=M^{n}$ with $n \geq 5$ and $K$ is an infinite field, then $\left|\Sigma^{\prime}(D)\right|=\infty$.

The paper consists of six sections, Section 1 is a review on almost pseudo-valuation domains, Section 2 is the general case, Section 3 is the case where $P=M^{2}$ or $P=M^{3}$, Section 4 is the case where $P=M^{4}$, Section 5 is the case where $P=M^{n}$ with $n \geq 5$, and Section 6 is examples.

\section{$\S 1$ Review}

In this section, we review a result in [M2] on semistar operations on almost pseudovaluation domains.

Let $I$ be an ideal of a domain $D$. If $a b \in I$ and $b \notin I$ imply $a^{n} \in I$ for some $n>0$ for each elements $a, b \in \mathrm{q}(D)$, then $I$ is called a strongly primary ideal of $D$, where $\mathrm{q}(D)$ denotes the quotient field of $D$. If each prime ideal of $D$ is strongly primary, then $D$ is called an almost pseudo-valuation domain (or an APVD). Every PVD is an almost pseudo-valuation domain. We confer Badawi-Houston $([\mathrm{BH}])$ for almost pseudovaluation domains.

(1.1) Let $D$ be an APVD, let $P$ be a maximal ideal of $D$, let $V=(P: P)$, and let $M$ be the maximal ideal of $V$.

(1) $\mathrm{F}^{\prime}(D)=\mathrm{F}(D) \cup\{\mathrm{q}(D)\}$.

(2) $D$ is a local ring, that is, $D$ has only one maximal ideal.

(3) If $D$ is not a valuation ring, then $V=P^{-1}$.

(4) The set of non-maximal prime ideals of $D$ coincides with the set of nonmaximal prime ideals of $V$, and $\operatorname{dim}(V)=\operatorname{dim}(D)$.

(5) The integral closure $\bar{D}$ of $D$ is a PVD with maximal ideal $M$.

(6) Let $T$ be an overring of $D$. Then either $T \supset V$ or $T \varsubsetneqq V$.

(7) Let $\Sigma_{1}^{\prime}=\left\{* \in \Sigma^{\prime}(D) \mid D^{*} \supset V\right\}$. Then there exists a canonical bijection from $\Sigma^{\prime}(V)$ onto $\Sigma_{1}^{\prime}$.

(8) Let $\Sigma_{2}^{\prime}=\left\{* \in \Sigma^{\prime}(D) \mid D^{*} \varsubsetneqq V\right\}$. Then $\Sigma^{\prime}(D)=\Sigma_{1}^{\prime} \cup \Sigma_{2}^{\prime}$. 
(9) If $\left|\Sigma^{\prime}(D)\right|<\infty$, then $\operatorname{dim}(D)<\infty, V=\bar{D}, V$ is a finitely generated $D$ module, and $V / M$ is a simple extension ficld of $D / P$ with $[V / M: D / P]<\infty$.

(1.2) Let $D$ be an APVD which is not a PVD, let $P$ be the maximal ideal of $D$, and let $V=(P: P)$. Assume that $\operatorname{dim}(D)<\infty$, and let $\left\{T_{\lambda} \mid \lambda \in \Lambda\right\}$ be the set of overrings $T$ of $D$ with $T \varsubsetneqq V$. Let $\Sigma_{1}^{\prime}$ be the set of semistar operations $*$ on $D$ such that $D^{*} \supset V$, and let $\Sigma_{2}^{\prime}$ be the set of semistar operations $*$ on $D$ such that $D^{*} \varsubsetneqq V$. Then we have

(1) $\Sigma^{\prime}(D)=\Sigma_{1}^{\prime} \cup \Sigma_{2}^{\prime}$.

(2) $\left|\Sigma^{\prime}(V)\right|<\infty$.

(3) $\left|\Sigma_{1}^{\prime}\right|=\left|\Sigma^{\prime}(V)\right|$.

(4) There exists a canonical bijection from the disjoint union $\bigcup_{\lambda} \Sigma\left(T_{\lambda}\right)$ onto $\Sigma_{2}^{\prime}$.

\section{$\S 2$ The general case}

Throughout the paper but the final $\S 6$, let $D$ be an APVD which is not a PVD, let $P$ be the maximal ideal of $D$, let $V=(P: P)$, let $M$ be the maximal ideal of $V$, let $v$ be a valuation belonging to $V$, and set $V / M=K$. We assume that $v$ is $Z$-valued and that $K=(D+M) / M$, and let $\left\{\alpha_{i} \mid i \in \mathcal{I}\right\}=\mathcal{K}$ be a system of complete representatives of $V$ modulo $M$, where $\{0,1\} \subset\left\{\alpha_{i} \mid i \in \mathcal{I}\right\} \subset D$.

Note: Let $V$ be a $Z$-valued valuation domain of the form $K+M$, where $K$ is a field and $M$ is the maximal ideal of $V$. Let $k$ be a subfield of $K$, and let $D=k+M$. Assume that $\operatorname{dim}(D)<\infty$, and $K$ is a simple extension of $k$ with finite degree. Then $|\Sigma(D)|$ need not be finite ([M3]).

There exists $\pi \in V$ such that $M=\pi V$. Then we have $v(\pi)=1$.

We have $V=P: P=P^{-1}$, where $P: P$ denotes $\{x \in \mathrm{q}(D) \mid x P \subset P\}$ and $P^{-1}$ denotes $D: P$.

Let $I, J \in \mathrm{F}(D)$. If there exists $x \in \mathrm{q}(D)$ such that $x J=I$, then $I$ and $J$ are said to be similar, and is denoted by $I \sim J$. For each $I \in \mathrm{F}(D)$, set $\{J \in \mathrm{F}(D) \mid J \sim I\}=$ $\operatorname{cl}(I)$.

Let $\Sigma_{1}^{\prime}$ be the set of semistar operations $*$ on $D$ such that $D^{*} \supset V$, and let $\Sigma_{2}^{\prime}$ be the set of semistar operations on $D$ such that $D^{*} \varsubsetneqq V$. We can apply (1.1) and (1.2) for $D$.

(2.1) Let $x \in \mathrm{q}(D)-\{0\}$, and let $k$ be a positive integer with $k>v(x)$. Then $x$ can be expressed uniquely as $x=\alpha_{l} \pi^{l}+\alpha_{l+1} \pi^{l+1}+\cdots+\alpha_{k-1} \pi^{k-1}+\pi^{k} a$, where $l=v(x)$ and each $\alpha_{i} \in \mathcal{K}$ with $\alpha_{l} \neq 0$ and $a \in V$.

(2.2) There exists a positive integer $n \geq 2$ uniquely so that $P=M^{n}$.

Proof. Set $\min \{v(x) \mid x \in P\}=n$. Since $P V=P$, we have $P=M^{n}$. If $n=1$, then $D$ is a valuation ring; a contradiction.

For every subset $A \subset \mathrm{q}(D)$, we denote by $(A)$ the $D$-submodule of $\mathrm{q}(D)$ generated by $A$. If $P=M^{n}$, then we have $P=\left(\pi^{n}, \pi^{n+1}, \cdots, \pi^{2 n-2}, \pi^{2 n-1}\right)$, and $V=\left(1, \pi, \cdots, \pi^{n-1}\right)$. 
(2.3) (1) We have $P^{v}=P$, where $P^{v}$ denotes $\left(P^{-1}\right)^{-1}$.

(2) The set $\operatorname{Spec}(D)$ of prime ideals of $D$ is $\{P,(0)\}$.

(2) follows from $(1.1)(4)$.

The mapping $I \longmapsto I^{v}$ from $\mathrm{F}(D)$ to $\mathrm{F}(D)$ is a star operation on $D$, and is called the $v$-operation. The identity mapping $d$ from $\mathrm{F}(D)$ to $\mathrm{F}(D)$ is a star operation on $D$, and is called the d-operation.

We note that $V$ is a divisorial fractional ideal of $D$, that is, $V^{\mathbf{v}}=V$. Each star operation on $D$ can be uniquely extended to a semistar operation on $D$. Also let $D^{\prime}$ be an overring of $D$. Then there exists a canonical mapping $\delta$ from $\Sigma^{\prime}\left(D^{\prime}\right)$ to $\Sigma^{\prime}(D)$, and is called the descent mapping. And $\delta$ is an injective mapping.

We have $\left|\Sigma_{1}^{\prime}\right|=\left|\Sigma^{\prime}(V)\right|=2$.

If $P=M^{2}$, then we have

$\{I \in \mathrm{F}(D) \mid D \subset I \subset V\}=\{(1),(1, \pi)\}$.

(2.4) Example Assume that $P=M^{3}$. Set $(1)=I_{0},\left(1, \pi^{2}\right)=I_{0,2},\left(1, \pi, \pi^{2}\right)=$ $I_{0,1,2}$, and $\left(1, \pi+\alpha \pi^{2}\right)=I_{0,1}^{\alpha}$, where $\alpha \in \mathcal{K}$. Then we have

$\{I \in \mathrm{F}(D) \mid D \subset I \subset V\}=\left\{I_{0}, I_{0,2}, I_{0,1,2}\right\} \cup\left\{I_{0,1}^{\alpha} \mid \alpha \in \mathcal{K}\right\}$

Proof. Because, $\{v(x) \mid x \in I-P\}$ is either $\{0\}$ or $\{0,1\}$ or $\{0,2\}$ or $\{0,1,2\}$.

(2.5) Example Assume that $P=M^{4}$. For every element $\alpha_{i} \in \mathcal{K}$, set

$(1)=I_{0}$,

$\left(1, \pi+\alpha_{1} \pi^{2}+\alpha_{2} \pi^{3}\right)=I_{0,1}^{\alpha_{1}, \alpha_{2}}$,

$\left(1, \pi^{2}+\alpha_{1} \pi^{3}\right)=I_{0,2}^{\alpha_{1}}$,

$\left(1, \pi^{3}\right)=I_{0,3}$

$\left(1, \pi+\alpha_{1} \pi^{3}, \pi^{2}+\alpha_{2} \pi^{3}\right)=I_{0,1.2}^{\alpha_{1}, \alpha_{2}}$,

$\left(1, \pi+\alpha_{1} \pi^{2}, \pi^{3}\right)=I_{0,1,3}^{\alpha_{1}}$,

$\left(1, \pi^{2}, \pi^{3}\right)=I_{0,2,3}$

$\left(1, \pi, \pi^{2}, \pi^{3}\right)=I_{0,1,2,3}$.

Then we have

$\{I \in \mathrm{F}(D) \mid D \subset I \subset V\}=\left\{I_{0}, I_{0,1}^{\alpha_{1}, \alpha_{2}}, I_{0,2}^{\alpha_{1}}, I_{0,3}, I_{0,1,2}^{\alpha_{1}, \alpha_{2}}, I_{0,1,3}^{\alpha_{1}}, I_{0,2,3}, I_{0,1,2,3} \mid\right.$ each $\left.\alpha_{i} \in \mathcal{K}\right\}$.

Proof. Let $I$ be a fractional ideal of $D$ such that $D \subset I \subset V$. Let $\tau=\{v(x) \mid x \in$ $I-P\}$, say let $\tau=\{0,1,3\}$. Then $I$ contains elements $a, b \in V$ of the form $a=$ $\pi+\alpha_{1} \pi^{2}, b=\pi^{3}$, where $\alpha_{1} \in \mathcal{K}$. We have $I \supset(1, a, b)$. Let $I \ni x=\beta_{0}+\beta_{1} \pi+\beta_{2} \pi^{2}+$ $\beta_{3} \pi^{3}+p$, where each $\beta_{i} \in \mathcal{K}$ and $p \in P$. We have $x=\beta_{0}+\beta_{1} a+\beta_{3} b+\beta^{\prime} \pi^{2}+p^{\prime}$ for some $\beta^{\prime} \in \mathcal{K}$ and $p^{\prime} \in P$. By the choice of $\tau$, we have $\beta^{\prime}=0$. Hence $I=(1, a, b)$.

(2.6) Example Assume that $P=M^{5}$. For every element $\alpha_{i} \in \mathcal{K}$, set

(1) $=I_{0}$,

$\left(1, \pi+\alpha_{1} \pi^{2}+\alpha_{2} \pi^{3}+\alpha_{3} \pi^{4}\right)=I_{0,1}^{\alpha_{1}, \alpha_{2}, \alpha_{3}}$,

$\left(1, \pi^{2}+\alpha_{1} \pi^{3}+\alpha_{2} \pi^{4}\right)=I_{0,2}^{\alpha_{1}, \alpha_{2}}$, 


$$
\begin{aligned}
& \left(1, \pi^{3}+\alpha_{1} \pi^{4}\right)=I_{0,3}^{\alpha_{1}}, \\
& \left(1, \pi^{4}\right)=I_{0,4}, \\
& \left(1, \pi+\alpha_{1} \pi^{3}+\alpha_{2} \pi^{4}, \pi^{2}+\alpha_{3} \pi^{3}+\alpha_{4} \pi^{4}\right)=I_{0,1,2}^{\alpha_{1}, \alpha_{2}, \alpha_{3}, \alpha_{4}}, \\
& \left(1, \pi+\alpha_{1} \pi^{2}+\alpha_{2} \pi^{4}, \pi^{3}+\alpha_{3} \pi^{4}\right)=I_{0,1,3}^{\alpha_{1}, \alpha_{2}, \alpha_{3}} \\
& \left(1, \pi+\alpha_{1} \pi^{2}+\alpha_{2} \pi^{3}, \pi^{4}\right)=I_{0,1,4}^{\alpha_{1}, \alpha_{2}}, \\
& \left(1, \pi^{2}+\alpha_{1} \pi^{4}, \pi^{3}+\alpha_{2} \pi^{4}\right)=I_{0,2,3}^{\alpha_{1}, \alpha_{2}} \\
& \left(1, \pi^{2}+\alpha_{1} \pi^{3}, \pi^{4}\right)=I_{0,2,4}^{\alpha_{1}}, \\
& \left(1, \pi^{3}, \pi^{4}\right)=I_{0,3,4} \\
& \left(1, \pi+\alpha_{1} \pi^{4}, \pi^{2}+\alpha_{2} \pi^{4}, \pi^{3}+\alpha_{3} \pi^{4}\right)=I_{0,1,2,3}^{\alpha_{1}, \alpha_{2}, \alpha_{3}}, \\
& \left(1, \pi+\alpha_{1} \pi^{3}, \pi^{2}+\alpha_{2} \pi^{3}, \pi^{4}\right)=I_{0,1,2,4}^{\alpha_{1}, \alpha_{2}}, \\
& \left(1, \pi+\alpha_{1} \pi^{2}, \pi^{3}, \pi^{4}\right)=I_{0,1,3,4}^{\alpha_{1}}, \\
& \left(1, \pi^{2}, \pi^{3}, \pi^{4}\right)=I_{0,2,3,4} \\
& \left(1, \pi, \pi^{2}, \pi^{3}, \pi^{4}\right)=I_{0,1,2,3,4} . \\
& T,{ }^{4},
\end{aligned}
$$

Then we have

$\{I \in \mathrm{F}(D) \mid D \subset I \subset V\}=\left\{I_{0}, I_{0,1}^{\alpha_{1}, \alpha_{2}, \alpha_{3}}, I_{0,2}^{\alpha_{1}, \alpha_{2}} . I_{0,3}^{\alpha_{1}}, I_{0,4}, I_{0,1,2}^{\alpha_{1}, \alpha_{2}, \alpha_{3}, \alpha_{4}}, I_{0,1,3}^{\alpha_{1}, \alpha_{2}, \alpha_{3}}\right.$, $I_{0,1,4}^{\alpha_{1}, \alpha_{2}}, I_{0,2,3}^{\alpha_{1}, \alpha_{2}}, I_{0,2,4}^{\alpha_{1}}, I_{0,3,4}, I_{0,1,2,3}^{\alpha_{1}, \alpha_{2}, \alpha_{3}}, I_{0.1,2,4}^{\alpha_{1}, \alpha_{2}}, I_{0,1,3,4}^{\alpha_{1}}, I_{0,2,3,4}, I_{0,1,2,3,4} \mid$ each $\left.\alpha_{i} \in \mathcal{K}\right\}$.

Proof. Let $I$ be a fractional ideal of $D$ such that $D \subset I \subset V$. Let $\tau=\{v(x) \mid x \in$ $I-P\}$, say let $\tau=\{0,1,3\}$. Then $I$ contains elements $a, b \in V$ of the form $a=$ $\pi+\alpha_{1} \pi^{2}+\alpha_{2} \pi^{4}, b=\pi^{3}+\alpha_{3} \pi^{4}$, where $\alpha_{1}, \alpha_{2}, \alpha_{3} \in \mathcal{K}$. We have $I \supset(1, a, b)$. Let $I \ni x=\beta_{0}+\beta_{1} \pi+\beta_{2} \pi^{2}+\beta_{3} \pi^{3}+\beta_{4} \pi^{4}+p$, where each $\beta_{i} \in \mathcal{K}$ and $p \in P$. We have $x=\beta_{0}+\beta_{1} a+\beta_{3} b+\beta_{1}^{\prime} \pi^{2}+\beta_{2}^{\prime} \pi^{4}+p^{\prime}$ for some $\beta_{i}^{\prime} \in \mathcal{K}$ and $p^{\prime} \in P$. By the choice of $\tau$, we have $\beta_{1}^{\prime}=\beta_{2}^{\prime}=0$. Hence $I=(1, a, b)$.

Each subset $\tau$ of $\{0,1,2,3,4\}$ which contains 0 is called a type associated to $D$. We have the number 16 of associated types to $D$. The set of types has a canonical order so that $\{0\}$ is the minimal member and $\{0,1,2,3,4\}$ is the maximal member: $\{0\},\{0,1\},\{0,2\},\{0,3\},\{0,4\},\{0,1,2\},\{0,1,3\},\{0,1,4\},\{0,2,3\},\{0,2,4\},\{0,3,4\}$, $\{0,1,2,3\},\{0,1,2,4\},\{0,1,3,4\},\{0,2,3,4\},\{0,1,2,3,4\}$. Let $\tau$ be a type associated to $D$, say let $\tau=\{0,1,2\}$. Then the tuple $\left\langle\alpha_{1}, \alpha_{2}, \alpha_{3}, \alpha_{4}>\right.$ of elements in $\mathcal{K}$ is called a system of parameters of $\tau$ (or, a system of parametcrs associated to $\tau$ ), and 4 is called the length of the system of parameters $\left\langle\alpha_{1}, \alpha_{2}, \alpha_{3}, \alpha_{4}\right\rangle$. The pair $\left.<0,1,2 ; \alpha_{1}, \alpha_{2}, \alpha_{3}, \alpha_{4}\right\rangle=\sigma$ of $\tau$ and $\left\langle\alpha_{1}, \alpha_{2}, \alpha_{3}, \alpha_{4}>\right.$ is called a data of $\tau$ (or, a data associated to $\tau$ ). Set $1=f_{1}^{\sigma}, \pi+\alpha_{1} \pi^{3}+\alpha_{2} \pi^{4}=f_{2}^{\sigma}, \pi^{2}+\alpha_{3} \pi^{3}+\alpha_{4} \pi^{4}=f_{3}^{\sigma}$. Then the tuple $<f_{1}^{\sigma}, f_{2}^{\sigma}, f_{3}^{\sigma}>$ is called an associated system of generators to $\sigma$. The fractional ideal $\left(f_{1}^{\sigma}, f_{2}^{\sigma}, f_{3}^{\sigma}\right)=I$ is called associated to $\sigma$, and is denoted by $I^{\sigma}$. The tuple $<f_{1}^{\sigma}, f_{2}^{\sigma}, f_{3}^{\sigma}>$ is also called a canonical system of generators for $I$ associated to $\sigma$.

Assume that $P=M^{n}$ for a positive integer $n \geq 2$. We confer the previous examples. Each subset $\tau$ of $\{0,1,2, \cdots, n-1\}$ which contains 0 is called a type associated to $D$. We have the number $2^{n-1}$ of associated types to $D$. The set of types has a canonical order so that $\{0\}=\tau_{1}$ is the minimal member and $\{0,1, \cdots, n-1\}=$ $\tau_{2^{n-1}}$ is the maximal member. Let $\tau=\left\{0, k_{1}, \cdots, k_{m}\right\}$ be a type associated to $D$ with $0<k_{1}<\cdots<k_{m}$. We can define a system of parameters $<\alpha_{1}, \cdots, \alpha_{l}>$ of $\tau$. It is a tuple of elements in $\mathcal{K}$. The pair $\left\langle 0, k_{1}, \cdots, k_{m} ; \alpha_{1}, \cdots, \alpha_{l}\right\rangle=\sigma$ of $\tau$ and 
$\left\langle\alpha_{1}, \cdots, \alpha_{l}\right\rangle$ is called a data of $\tau$ (or, a data associated to $\tau$ ). We can define an associated system $<f_{1}^{\sigma}, f_{2}^{\sigma}, \cdots, f_{m+1}^{\sigma}>$ of generators to $\sigma$. It is a tuple of elements in $V$. We donote also $f_{i}^{\sigma}=f_{i}(\sigma)$. The fractional ideal $\left(f_{1}^{\sigma}, f_{2}^{\sigma}, \cdots, f_{m+1}^{\sigma}\right)=I$ is said to be associated to $\sigma$, and is denoted by $I^{\sigma}$ (or, by $I(\sigma)$ ). The tuple $<f_{1}^{\sigma}, f_{2}^{\sigma}, \cdots, f_{m+1}^{\sigma}>$ is also called a canonical system of generators for $I$ associated to $\sigma$.

(2.7) Proposition Assume that $P=M^{n}$ with $n \geq 2$. Then we have $\{I \in \mathrm{F}(D) \mid D \subset I \subset V\}=\left\{I\left(\sigma_{1}\right), \cdots, I\left(\sigma_{2^{n-1}}\right) \mid\right.$ each $\sigma_{i}$ is a data associated to the type $\tau_{i}$ for $\left.1 \leq i \leq 2^{n-1}\right\}$.

$\S 3$ The case where $P=M^{2}$ or $P=M^{3}$

(3.1) Proposition Assume that $P=M^{2}$. Then we have $|\Sigma(D)|=1$ and $\left|\Sigma^{\prime}(D)\right|=3$.

Proof. Since $\pi^{2}(1, \pi)=P$, each $I \in \mathrm{F}(D)$ is divisorial. It follows that $|\Sigma(D)|=1$.

Let $T$ be an overring of $D$ with $V \supsetneqq T \supsetneqq D$, and take $t \in T-D$. There may arise the following two cases: (1) $v(t)=1$, and (2) $v(t)=0$.

Case (1): We may assume that $t=\pi+p$ for some $p \in P$. Hence we have $T \ni \pi$, and hence $T=V$; a contradiction.

Case (2): We may assume that $t=1+\alpha \pi+p$ for some $\alpha \in \mathcal{K}$ and $p \in P$. Since $t \notin D$, we have $\alpha \pi \in T-D$. Case (1) implies that $T=V$; a contradiction.

We will apply (1.2). Since $\left|\Sigma_{1}^{\prime}\right|=2$, we have $\left|\Sigma^{\prime}(D)\right|=2+|\Sigma(D)|=3$.

Throughout the rest of the section, assume that $P=M^{3}$.

(3.2) We have

$\{T \mid T$ is an overring of $D$ with $T \varsubsetneqq V\}=\left\{D, D+M^{2}\right\}$.

Proof. Let $T$ be an overring of $D$ with $V \supsetneqq T \supsetneqq D$, and take $t \in T-D$. The proof of (3.1) shows that, if $T \supsetneqq D+M^{2}$ then $T=V$. There may arise the following three cases: (1) $v(t)=2,(2) v(t)=1$, and (3) $v(t)=0$.

Case (1): We may assume that $t=\pi^{2}+p$ for some $p \in P$. Hence we have $T \ni \pi^{2}$, and $T \supset D+M^{2}$.

Case (2): We may assume that $t=\pi+\alpha \pi^{2}+p$ for some $\alpha \in \mathcal{K}$ and $p \in P$. Since $T \ni t^{2}$, we have $T \ni \pi^{2}$, and hence $T \supset D+M^{2}$.

Case (3): We may assume that $t=1+\alpha_{1} \pi+\alpha_{2} \pi^{2}+p$ for some $\alpha_{1}, \alpha_{2} \in \mathcal{K}$ and $p \in P$. Since $t \notin D$, we have $\alpha_{1} \pi+\alpha_{2} \pi^{2} \in T-D$. Cases 1 and 2 imply that $T \supset D+M^{2}$.

(3.3) (1) $I_{0,2}$ and $I_{0,1}^{\alpha}$ are incomparable for each $\alpha \in \mathcal{K}$.

(2) $I_{0,1}^{\alpha} \subset I_{0,1}^{\beta}$ if and only if $\alpha=\beta$.

Proof. (2) Assume that $I_{0,1}^{\alpha} \subset I_{0,1}^{\beta}$. Then $\pi+\alpha \pi^{2} \in\left(1, \pi+\beta \pi^{2}\right)$ implies $\pi+\alpha \pi^{2}=\left(\pi+\beta \pi^{2}\right)+p$ for some $p \in P$. Hence $\alpha=\beta$. 
(3.4) (1) Each two in $\left\{I_{0}, I_{0,2}, I_{0,1,2}, I_{0,1}^{\alpha}\right\}$ are not similar for each $\alpha \in \mathcal{K}$.

(2) $I_{0,1}^{\alpha}$ and $I_{0,1}^{\beta}$ are similar for each $\alpha, \beta \in \mathcal{K}$.

Proof. (2) Set $1+\alpha \pi+\alpha^{2} \pi^{2}=x$. Then we have $x(1, \pi)=\left(1, \pi+\alpha \pi^{2}\right)$.

(3.5) Let $*$ be a star operation on $D$. Then $\left(I_{0,2}\right)^{*}$ is either $I_{0,2}$ or $V$, and $\left(I_{0,1}^{0}\right)^{*}$ is either $I_{0,1}^{0}$ or $V$.

Proof. Since $V$ is a divisorial fractional ideal of $D$, we have $\left(I_{0,2}\right)^{*} \subset V$ and $\left(I_{0,1}^{0}\right)^{*} \subset V$.

(3.6) (1) If we set $\left(I_{0,2}\right)^{*_{1}}=I_{0,2}$ and $\left(I_{0,1}^{0}\right)^{*_{1}}=I_{0,1}^{0}$, then there is determined a unique mapping $*_{1}$ from $\mathrm{F}(D)$ to $\mathrm{F}(D)$.

(2) If we set $\left(I_{0,2}\right)^{*_{2}}=I_{0,2}$ and $\left(I_{0,1}^{0}\right)^{*_{2}}=V$, then there is determined a unique mapping $*_{2}$ from $\mathrm{F}(D)$ to $\mathrm{F}(D)$.

(3) If we set $\left(I_{0,2}\right)^{*_{3}}=V$ and $\left(I_{0,1}^{0}\right)^{*_{3}}=I_{0,1}^{0}$, then there is determined a unique mapping $*_{3}$ from $\mathrm{F}(D)$ to $\mathrm{F}(D)$.

(4) If we set $\left(I_{0,2}\right)^{*_{4}}=V$ and $\left(I_{0,1}^{0}\right)^{*_{4}}=V$, then there is determined a unique mapping $*_{4}$ from $\mathrm{F}(D)$ to $\mathrm{F}(D)$.

For, each element $I \in \mathrm{F}(D)$ is similar to one and only one in $\left\{I_{0}, I_{0,2}, I_{0,1,2}, I_{0,1}^{0}\right\}$.

(3.7) (1) $*_{1}$ is a star operation on $D$, and $*_{1}=\mathrm{d}$.

(2) $*_{2}$ is a star operation on $D$.

(3) $*_{3}$ is not a star operation on $D$.

(4) $*_{4}$ is a star operation on $D$, and $*_{4}=v$.

Proof. (2) For each $x \in \mathrm{q}(D)-\{0\}$, we have $(x)^{*_{2}}=(x)$.

For each $x \in \mathrm{q}(D)-\{0\}$ and each $I \in \mathrm{F}(D)$, we have $(x I)^{*_{2}}=x I^{*_{2}}$.

For each $I \in \mathrm{F}(D)$, we have $I \subset I^{*_{2}}$.

For each $I \in \mathrm{F}(D)$, we have $\left(I^{*_{2}}\right)^{*_{2}}=I^{*_{2}}$.

Let $I_{1}, I_{2} \in \mathrm{F}(D)$ with $I_{1} \subset I_{2}$. To prove $I_{1}^{*_{2}} \subset I_{2}^{*_{2}}$, it is sufficient to show that, if $x I_{0,1}^{0} \subset J$ then $x V \subset J$ for each $x \in \mathrm{q}(D)-\{0\}$ and each $J \in\left\{I_{0}, I_{0,2}, I_{0,1,2}\right\}$.

(3) Set $\pi+\pi^{2}=x$. Then we have $x\left(1, \pi^{2}\right) \subset\left(1, \pi+\pi^{2}\right)$ and $x V \not \subset\left(1, \pi+\pi^{2}\right)$.

(3.8) Proposition Assume that $P=M^{3}$. Then we have $|\Sigma(D)|=3$ and $\left|\Sigma^{\prime}(D)\right|=6$.

Proof. It follows that $\Sigma(D)=\left\{d, v, *_{2}\right\}$, and that $|\Sigma(D)|=3$. We can apply (3.1) for $D^{\prime}=D+M^{2}$. Then we have

$\left|\Sigma_{2}^{\prime}\right|=|\Sigma(D)|+\left|\Sigma\left(D+M^{2}\right)\right|=3+1=4$.

Since $\left|\Sigma^{\prime}(V)\right|=2$, it follows that

$\left|\Sigma^{\prime}(D)\right|=\left|\Sigma_{1}^{\prime}\right|+\left|\Sigma_{2}^{\prime}\right|=2+4=6$. 


\section{$\S 4$ The case where $P=M^{4}$}

In this section, we assume that $P=M^{4}$.

(4.1) Proposition If $K$ is a finite field, then we have $|\Sigma(D)|<\infty$.

Proof. Let

$X=\left\{I_{0}, I_{0,1}^{\alpha_{1}, \alpha_{2}}, I_{0,2}^{\alpha_{1}}, I_{0,3}, I_{0.1,2}^{\alpha_{1}, \alpha_{2}}, I_{0,1,3}^{\alpha_{1}}, I_{0,2,3}, I_{0,1,2,3} \mid \operatorname{cach} \alpha_{i} \in \mathcal{K}\right\}$.

Then $X$ is a finite set. Let * be a star operation on $D$. Since $V$ is a divisorial fractional ideal of $D$, we have $D \subset I^{*} \subset V$ for each $I \in X$. If we set $I^{*}=g_{*}(I)$, each element $* \in \Sigma(D)$ gives rise to an element $g_{*} \in X^{X}$, where $X^{X}$ is the set of mappings from $X$ to $X$. And the mapping $g: * \longmapsto g_{*}$ from $\Sigma(D)$ to $X^{X}$ is an injection.

(4.2) Let $\alpha_{1}, \alpha_{2}, \beta_{1}, \beta_{2} \in \mathcal{K}$.

$I_{0,1}^{\alpha_{1}, \alpha_{2}} \subset I_{0,1}^{\beta_{1}, \beta_{2}}$ if and only if $\alpha_{1}=\beta_{1}, \alpha_{2}=\beta_{2}$.

$I_{0,2}^{\alpha_{1}} \subset I_{0,2}^{\beta_{1}}$ if and only if $\alpha_{1}=\beta_{1}$.

$I_{0,1,2}^{\alpha_{1}, \alpha_{2}} \subset I_{0,1,2}^{\beta_{1}, \beta_{2}}$ if and only if $\alpha_{1}=\beta_{1}, \alpha_{2}=\beta_{2}$.

$I_{0,1,3}^{\alpha_{1}} \subset I_{0,1,3}^{\beta_{1}, 1}$ if and only if $\alpha_{1}=\beta_{1}$.

Proof. For instance, assume that $I_{0,1,2}^{\alpha_{1}, \alpha_{2}} \subset I_{0,1,2}^{\beta_{1}, \beta_{2}}$. Then we have $\pi+\alpha_{1} \pi^{3}=\left(\pi+\beta_{1} \pi^{3}\right)+\left(\pi^{2}+\beta_{3} \pi^{3}\right) p_{1}+p_{2}$

for some $p_{i} \in P$. Hence $\alpha_{1}=\beta_{1}$. We have $\pi^{2}+\alpha_{2} \pi^{3}=\left(\pi^{2}+\beta_{2} \pi^{3}\right)+p_{3}$ for some $p_{3} \in P$. Hence $\alpha_{2}=\beta_{2}$.

(4.3) (1) $I_{0,1}^{\alpha_{1}, \alpha_{2}} \sim I_{0,1}^{\beta_{1}, \beta_{2}}$ if and only if $\beta_{1}^{2}-\beta_{2} \equiv \alpha_{1}^{2}-\alpha_{2}(\bmod P)$.

(2) $I_{0,2}^{\alpha} \sim I_{0,2}^{\beta}$ if and only if $\alpha=\beta$.

(3) $I_{0,1,2}^{\alpha_{1}, \alpha_{2}} \sim I_{0,1,2}^{\beta_{1}, \beta_{2}}$ for each $\alpha_{1}, \alpha_{2}, \beta_{1}, \beta_{2} \in \mathcal{K}$.

(4) $I_{0,1,3}^{\alpha} \sim I_{0,1,3}^{\beta}$ for each $\alpha, \beta \in \mathcal{K}$.

Proof. (1) Assume that $\beta_{1}^{2}-\beta_{2} \equiv \alpha_{1}^{2}-\alpha_{2}(\bmod P)$. Set $1+\left(\pi+\beta_{1} \pi^{2}+\right.$ $\left.\beta_{2} \pi^{3}\right)\left(\beta_{1}-\alpha_{1}\right)=x$. Then we have $x I_{0,1}^{\alpha_{1}, \alpha_{2}}=I_{0,1}^{\beta_{1}, \beta_{2}}$.

(3) Set $1-\alpha_{2} \pi-\alpha_{1} \pi^{2}=x$. Then we have $x I_{0,1,2}^{\alpha_{1}, \alpha_{2}}=I_{0,1,2}^{0,0}$.

(4) Set $1-\alpha \pi=x$. Then we have $x I_{0,1,3}^{\alpha}=I_{0,1,3}^{0}$.

(4.4) Each two in $\left\{I_{0}, I_{0,1}^{\alpha_{1}, \alpha_{2}}, I_{0,2}^{\alpha_{3}}, I_{0,3}, I_{0,1.2}^{\alpha_{4}, \alpha_{5}}, I_{0,1,3}^{\alpha_{6}}, I_{0,2,3}, I_{0,1,2,3}\right\}$ are not similar for each $\alpha_{i} \in \mathcal{K}$.

Proof. For instance, suppose that there exists $x \in \mathrm{q}(D)$ so that $I_{0,1}^{\alpha_{1}, \alpha_{2}}=x I_{0,1,3}^{\alpha_{6}}$. We may assume that $x=1+\beta_{1} \pi+\beta_{2} \pi^{2}+\beta_{3} \pi^{3}+p$ for some $\beta_{i} \in \mathcal{K}$ and $p \in P$. Then $x \pi^{3} \in I_{0.1}^{\alpha_{1}, \alpha_{2}}$ implies a contradiction.

(4.5) Let $x \in \mathrm{q}(D)-\{0\}$.

(1) $x I_{0,1}^{\alpha_{1}, \alpha_{2}} \subset I_{0}$ implies $x V \subset I_{0}$.

(2) $x I_{0,1,2}^{\alpha_{1}, \alpha_{2}} \subset I_{0}$ implies $x V \subset I_{0}$. 
(3) $x I_{0,1,3}^{\alpha} \subset I_{0}$ implies $x V \subset I_{0}$.

(4) $x I_{0,1}^{\alpha_{1}, \alpha_{(2)}} \subset I_{0,1}^{\alpha_{1}, \alpha_{2}}$ with $\alpha_{(1)}^{2}-\alpha_{(2)} \not \equiv \alpha_{1}^{2}-\alpha_{2}(\bmod P)$ implies $x V \subset I_{0,1}^{\alpha_{1}, \alpha_{2}}$.

(5) $x I_{0,1}^{\alpha_{1}, \alpha_{2}} \subset I_{0,2}^{\beta}$ implies $x V \subset I_{0,2}^{\beta}$.

(6) $x I_{0,1}^{\alpha_{1}, \alpha_{2}} \subset I_{0,3}$ implies $x V \subset I_{0,3}$.

(7) $x I_{0,1}^{\alpha_{1}, \alpha_{2}} \subset I_{0,2,3}$ implies $x V \subset I_{0,2,3}$.

(8) $x I_{0,1,2}^{\alpha_{1}, \alpha_{2}} \subset I_{0,1}^{\beta_{1}, \beta_{2}}$ implies $x V \subset I_{0,1}^{\beta_{1}, \beta_{2}}$.

(9) $x I_{0,1,3}^{\alpha_{1}} \subset I_{0,1}^{\beta_{1}, \beta_{2}}$ implies $x V \subset I_{0,1}^{\beta_{1}, \beta_{2}}$.

(10) $x I_{0,1,2}^{\alpha_{1}, \alpha_{2}} \subset I_{0,2}^{\beta}$ implies $x V \subset I_{0,2}^{\beta}$.

(11) $x I_{0,1,3}^{\alpha} \subset I_{0,2}^{\beta}$ implies $x V \subset I_{0,2}^{\beta}$.

(12) $x I_{0,1,2}^{\alpha_{1}, \alpha_{2}} \subset I_{0,3}$ implies $x V \subset I_{0,3}$.

(13) $x I_{0,1,3}^{\alpha} \subset I_{0,3}$ implies $x V \subset I_{0.3}$.

(14) $x I_{0,1,2}^{\alpha_{1}, \alpha_{2}} \subset I_{0,2,3}$ implies $x V \subset I_{0,2,3}$.

(15) $x I_{0,1,3}^{\alpha} \subset I_{0,2,3}$ implies $x V \subset I_{0,2,3}$.

Proof. (4) We may assume that $v(x)=0$. Then we may assume that $x=1+$ $\left(\pi+\alpha_{1} \pi^{2}+\alpha_{2} \pi^{3}\right) \alpha+p$ for some $\alpha \in \mathcal{K}$ and $p \in P$. Then $x\left(\pi+\alpha_{(1)} \pi^{2}+\alpha_{(2)} \pi^{3}\right) \in I_{0,1}^{\alpha_{1}, \alpha_{2}}$ implies $\alpha_{(1)}^{2}-\alpha_{(2)} \equiv \alpha_{1}^{2}-\alpha_{2}(\bmod P)$; a contradiction. It follows that $x \in M^{4}$, and hence $x V \subset I_{0,1}^{\alpha_{1}, \alpha_{2}}$.

(4.6) Fix a data $\left\langle 0,1 ; \alpha_{(1)}, \alpha_{(2)}\right\rangle$, and set $\left(I_{0,1}^{\alpha_{(1)}, \alpha_{(2)}}\right)^{*}=V$. For $I_{0,1}^{\alpha_{1}, \alpha_{2}}$ with $\alpha_{1}^{2}-\alpha_{2} \not \equiv \alpha_{(1)}^{2}-\alpha_{(2)}(\bmod P)$, set $\left(I_{0,1}^{\alpha_{1}, \alpha_{2}}\right)^{*}=I_{0,1}^{\alpha_{1}, \alpha_{2}}$. For each $\beta_{1}, \beta_{2} \in \mathcal{K}$, set $\left(I_{0,2}^{\beta_{1}}\right)^{*}=I_{0,2}^{\beta_{1}},\left(I_{0,1,2}^{\beta_{1}, \beta_{2}}\right)^{*}=V,\left(I_{0,1,3}^{\beta_{1}}\right)^{*}=V$, and set $\left(I_{0,3}\right)^{*}=I_{0,3},\left(I_{0,2,3}\right)^{*}=I_{0,2,3}$.

Then we have

(1) There is determined a unique mapping * from $\mathrm{F}(D)$ to $\mathrm{F}(D)$.

(2) For each $x \in \mathrm{q}(D)-\{0\}$, we have $(x)^{*}=(x)$.

(3) For each $x \in \mathrm{q}(D)-\{0\}$ and each $I \in \mathrm{F}(D)$, we have $(x I)^{*}=x I^{*}$.

(4) For each $I \in \mathrm{F}(D)$, we have $I \subset I^{*}$.

(5) For each $I \in \mathrm{F}(D)$, we have $\left(I^{*}\right)^{*}=I^{*}$.

(6) For each $I_{1}, I_{2} \in \mathrm{F}(D)$ with $I_{1} \subset I_{2}$, we have $I_{1}^{*} \subset I_{2}^{*}$.

(6) follows from (4.5).

(4.7) Proposition Assume that $P=M^{4}$ and $K$ is an infinite field. Then we have $|\Sigma(D)|=\infty$ and $\left|\Sigma^{\prime}(D)\right|=\infty$.

Proof. Let $*_{\alpha_{(1)}, \alpha_{(2)}}$ be the star operation on $D$ determined in (4.6). If $I_{0,1}^{\alpha_{1}, \alpha_{2}} \nsim$ $I_{0,1}^{\beta_{1}, \beta_{2}}$, we have $*_{\alpha_{1}, \alpha_{2}} \neq *_{\beta_{1}, \beta_{2}}$. It follows that $|\Sigma(D)|=\infty$.

\section{$\S 5$ The case where $P=M^{n}$ with $n \geq 5$}

(5.1) Proposition Assume that $P=M^{5}$ and $K$ is a finite field. Then we have $|\Sigma(D)|<\infty$. 
Proof. Let

$X=\left\{I_{0}, I_{0,1}^{\alpha_{1}, \alpha_{2}, \alpha_{3}}, I_{0,2}^{\alpha_{1}, \alpha_{2}}, I_{0,3}^{\alpha_{1}}, I_{0,4}, I_{0,1,2}^{\alpha_{1}, \alpha_{2}, \alpha_{3}, \alpha_{4}}, I_{0,1,3}^{\alpha_{1}, \alpha_{2}, \alpha_{3}}, I_{0,1,4}^{\alpha_{1}, \alpha_{2}}, I_{0,2,3}^{\alpha_{1}, \alpha_{2}}, I_{0,2,4}^{\alpha_{1}}\right.$, $I_{0,3,4}, I_{0,1,2,3}^{\alpha_{1}, \alpha_{2}, \alpha_{3}}, I_{0,1,2,4}^{\alpha_{1}, \alpha_{2}}, I_{0,1,3,4}^{\alpha_{1}}, I_{0,2,3,4}, I_{0,1,2,3,4} \mid$ each $\left.\alpha_{i} \in \mathcal{K}\right\}$.

The similar argument to the proof of (4.1) completes the proof.

(5.2) Assume that $P=M^{5}$. Let $\alpha_{i}, \beta_{j} \in \mathcal{K}$ for each $i, j$.

$I_{0,1}^{\alpha_{1}, \alpha_{2}, \alpha_{3}} \subset I_{0,1}^{\beta_{1}, \beta_{2}, \beta_{3}}$ if and only if $\alpha_{i}=\beta_{i}$ for each $i$.

$I_{0,2}^{\alpha_{1}, \alpha_{2}} \subset I_{0,2}^{\beta_{1}, \beta_{2}}$ if and only if $\alpha_{i}=\beta_{i}$ for each $i$.

$I_{0,3}^{\alpha_{1}} \subset I_{0,3}^{\beta_{1}}$ if and only if $\alpha_{1}=\beta_{1}$.

$I_{0,1,2}^{\alpha_{1}, \alpha_{2}, \alpha_{3}, \alpha_{4}} \subset I_{0,1,2}^{\beta_{1}, \beta_{2}, \beta_{3}, \beta_{4}}$ if and only if $\alpha_{i}=\beta_{i}$ for each $i$.

$I_{0,1,3}^{\alpha_{1}, \alpha_{2}, \alpha_{3}} \subset I_{0,1,3}^{\beta_{1}, \beta_{2}, \beta_{3}}$ if and only if $\alpha_{i}=\beta_{i}$ for each $i$.

$I_{0,1,4}^{\alpha_{1}, \alpha_{2}} \subset I_{0,1,4}^{\beta_{1}, \beta_{2}}$ if and only if $\alpha_{i}=\beta_{i}$ for each $i$.

$I_{0,2,3}^{\alpha_{1}, \alpha_{2}} \subset I_{0,2,3}^{\beta_{1}, \beta_{2}}$ if and only if $\alpha_{i}=\beta_{i}$ for each $i$.

$I_{0,2,4}^{\alpha_{1}} \subset I_{0,2,4}^{\beta_{1}}$ if and only if $\alpha_{1}=\beta_{1}$.

$I_{0.1,2,3}^{\alpha_{1}, \alpha_{2}, \alpha_{3}} \subset I_{0.1,2,3}^{\beta_{1}, \beta_{2}, \beta_{3}}$ if and only if $\alpha_{i}=\beta_{i}$ for each $i$.

$I_{0,1,2,4}^{\alpha_{1}, \alpha_{2}} \subset I_{0,1,2,4}^{\beta_{1}, \beta_{2}}$ if and only if $\alpha_{i}=\beta_{i}$ for each $i$.

$I_{0,1,3,4}^{\alpha_{1}} \subset I_{0,1,3,4}^{\beta_{1}}$ if and only if $\alpha_{1}=\beta_{1}$.

Proof. For instance, assume that $I_{0,1,2}^{\alpha_{1}, \alpha_{2}, \alpha_{3}, \alpha_{4}} \subset I_{0,1,2}^{\beta_{1}, \beta_{2}, \beta_{3}, \beta_{4}}$. Then we have $\pi+\alpha_{1} \pi^{3}+\alpha_{2} \pi^{4}=\left(\pi+\beta_{1} \pi^{3}+\beta_{2} \pi^{4}\right)+\left(\pi^{2}+\beta_{3} \pi^{3}+\beta_{4} \pi^{4}\right) p_{1}+p_{2}$

for some $p_{i} \in P$. Hence $\alpha_{1}=\beta_{1}$ and $\alpha_{2}=\beta_{2}$. We have

$$
\pi^{2}+\alpha_{3} \pi^{3}+\alpha_{4} \pi^{4}=\left(\pi^{2}+\beta_{3} \pi^{3}+\beta_{4} \pi^{4}\right)+p_{3}
$$

for some $p_{3} \in P$. Hence $\alpha_{3}=\beta_{3}$ and $\alpha_{4}=\beta_{4}$.

(5.3) Assume that $P=M^{5}$. Then each two in $\left\{I_{0}, I_{0,1}^{\alpha_{1}, \alpha_{2}, \alpha_{3}}, I_{0,2}^{\alpha_{4}, \alpha_{5}}, I_{0,3}^{\alpha_{6}}, I_{0,4}\right.$, $I_{0,1,2}^{\alpha_{7}, \alpha_{8}, \alpha_{9}, \alpha_{10}}, I_{0,1,3}^{\alpha_{11}, \alpha_{12}, \alpha_{13}}, I_{0,1,4}^{\alpha_{14}, \alpha_{15}}, I_{0,2,3}^{\alpha_{16}, \alpha_{17}}, I_{0,2,4}^{\alpha_{18}}, I_{0,3,4}, I_{0,1,2,3}^{\alpha_{19}, \alpha_{20}, \alpha_{21}}, I_{0,1,2,4}^{\alpha_{22}, \alpha_{23}}, I_{0,1,3,4}^{\alpha_{24}}$, $\left.I_{0,2,3,4}, I_{0,1,2,3,4}\right\}$ are not similar for each $\alpha_{\imath} \in \mathcal{K}$.

Proof. Because the each two have distinct types.

(5.4) Proposition Assume that $P=M^{n}$ with $n \geq 6$ and $K$ is a finite field. Then we have $|\Sigma(D)|<\infty$.

The proof is similar to that of (5.1).

(5.5) Proposition Assume that $P=M^{n}$ with $n \geq 5$ and $K$ is an infinite field. Then we have $\left|\Sigma^{\prime}(D)\right|=\infty$.

Proof. Set $D+M^{4}=D^{\prime}$. Then $D^{\prime}$ is an APVD with maximal ideal $M^{4}$. $D^{\prime}$ is not a PVD. We have $\left|\Sigma\left(D^{\prime}\right)\right|=\infty$ by (4.7). Hence $\left|\Sigma^{\prime}\left(D^{\prime}\right)\right|=\infty$. Since the descent map $\delta$ from $\Sigma^{\prime}\left(D^{\prime}\right)$ to $\Sigma^{\prime}(D)$ is an injection, we have $\left|\Sigma^{\prime}(D)\right|=\infty$.

The proof of our Theorem is complete. 


\section{$\S 6$ Examples}

In this final section, $D$ does not necessarily denote an APVD which is not a PVD. We will apply our Theorem to some APVD's.

(6.1) Proposition Let $V$ be a rank one discrete valuation domain of the form $K+M$, where $K$ is a field and $M$ is the maximal ideal of $V$, and let $D=K+M^{n}$ for a positive integer $n \geq 2$. Then we have

(1) If $K$ is a finite field, then $|\Sigma(D)|<\infty$.

(2) If $n=2$, then $|\Sigma(D)|=1$ and $\left|\Sigma^{\prime}(D)\right|=3$, and if $n=3$, then $|\Sigma(D)|=3$ and $\left|\Sigma^{\prime}(D)\right|=6$.

(3) If $n=4$ and $K$ is an infinite field, then $|\Sigma(D)|=\infty$ and $\left|\Sigma^{\prime}(D)\right|=\infty$.

(4) If $n \geq 5$ and $K$ is an infinite field, then $\left|\Sigma^{\prime}(D)\right|=\infty$.

Proof. Then $D$ is an APVD which is not a PVD, $P=M^{n}$ is the maximal ideal of $D, V=(P: P)$, and $(D+M) / M=V / M$.

(6.2) Let $V=K[[X]]$ be the formal power series ring of a variable $X$ over a field $K$, let $M$ be the maximal ideal of $V$, and let $D=K+M^{n}$ for a positive integer $n \geq 2$. Then we have the same $(1) \sim(4)$ of $(6.1)$.

(6.3) Let $V$ be a rank one discrete valuation domain of the form $K+M$, where $K$ is a field and $M$ is the maximal ideal of $V$, and let $D=k+M^{n}$ for a positive integer $n \geq 2$ and for a subfield $k$ of $K$. Then, if $n \geq 4$ and $K$ is an infinite field, then $\left|\Sigma^{\prime}(D)\right|=\infty$.

Proof. Set $K+M^{n}=D^{\prime}$. Then $\left|\Sigma^{\prime}\left(D^{\prime}\right)\right|=\infty$ by (6.1). Since the descent map $\delta$ from $\Sigma^{\prime}\left(D^{\prime}\right)$ to $\Sigma^{\prime}(D)$ is an injection, we have $\left|\Sigma^{\prime}(D)\right|=\infty$.

\section{References}

[BH] A. Badawi and E. Houston, Powerful ideals, strongly primary ideals, almost pseudo-valuation domains, and conducive domains, Comm. Alg. 30(2002), 15911606.

[FL] M. Fontana and K. Loper, Kronecker function rings: a general approach, Ideal theoretic methods in commutative algebra, Lecture notes in Pure and Appl. Math., 220, Dekker, New York, 2001, 189-205.

[HK] F. Halter-Koch, Ideal Systems: An Introduction to Multiplicative Ideal Theory, Marcel Dekker, 1998.

[K] W. Krull, Beiträge zur Arithmetik kommutativer Integritätesbcreiche, Math. Zeit. 41(1936), 545-577. 
[M1] R. Matsuda, Note on the number of semistar-operations, Math. J. Ibaraki Univ. 31 (1999), 47-53.

[M2] R. Matsuda, Note on the number of semistar operations, VIII, Math. J. Ibaraki Univ. 37 (2005), 53-79.

[M3] R. Matsuda, Note on the number of semistar operations, VII, J. Commutative Algebra, to appear.

[M4] R. Matsuda, Integrally closed domains with a finite number of semistar operations, J. Commutative Algebra, to appear.

[M5] R. Matsuda, Semistar operations on a pseudo-valuation domain, J. Commutative Algebra, to appear.

[P] H. Prüfer, Untersufungen über Teilbarkeitseigenshaften in Körpern, J. reine angew. Math. 168(1932), 1-36. 